\title{
Omalizumab for Severe Asthma: Beyond Allergic Asthma
}

\author{
C. C. Loureiro, ${ }^{1,2}$ L. Amaral, ${ }^{3}$ J. A. Ferreira, ${ }^{4}$ R. Lima, ${ }^{5}$ C. Pardal, ${ }^{6}$ I. Fernandes, ${ }^{7}$ \\ L. Semedo, ${ }^{8,9}$ and A. Arrobas $\mathbb{D}^{10}$
}

${ }^{1}$ Pulmonology Unit, Hospitais da Universidade de Coimbra, Centro Hospitalar e Universitário de Coimbra, Coimbra, Portugal

${ }^{2}$ Centre of Pulmonology, Faculty of Medicine, University of Coimbra, Coimbra, Portugal

${ }^{3}$ Immunoallergology Department, Centro Hospitalar São João, Porto, Portugal

${ }^{4}$ Immunoallergology Department, Centro Hospitalar de Vila Nova de Gaia/Espinho (CHVNGE), Vila Nova de Gaia, Portugal

${ }^{5}$ Pulmonology Department, Centro Hospitalar de Vila Nova de Gaia/Espinho (CHVNGE), Vila Nova de Gaia, Portugal

${ }^{6}$ Pulmonology Department, Hospital Professor Doutor Fernando Fonseca, EPE, Amadora, Portugal

${ }^{7}$ Pulmonology Department, Hospital São Bernardo, Setúbal, Portugal

${ }^{8}$ Pulmonology Department, Hospital Santa Marta, Lisboa, Portugal

${ }^{9}$ NOVA Medical School, Lisbon, Portugal

${ }^{10}$ Pulmonology Unit, Hospital Geral, Centro Hospitalar e Universitário de Coimbra, Coimbra, Portugal

Correspondence should be addressed to A. Arrobas; ana.arrobas@gmail.com

Received 29 March 2018; Accepted 18 July 2018; Published 17 September 2018

Academic Editor: Enrico Heffler

Copyright (c) 2018 C. C. Loureiro et al. This is an open access article distributed under the Creative Commons Attribution License, which permits unrestricted use, distribution, and reproduction in any medium, provided the original work is properly cited.

\begin{abstract}
Different subsets of asthma patients may be recognized according to the exposure trigger and the frequency and severity of clinical signs and symptoms. Regarding the exposure trigger, generally asthma can be classified as allergic (or atopic) and nonallergic (or nonatopic). Allergic and nonallergic asthma are distinguished by the presence or absence of clinical allergic reaction and in vitro IgE response to specific aeroallergens. The mechanisms of allergic asthma have been extensively studied with major advances in the last two decades. Nonallergic asthma is characterized by its apparent independence from allergen exposure and sensitization and a higher degree of severity, but little is known regarding the underlying mechanisms. Clinically, allergic and nonallergic asthma are virtually indistinguishable in exacerbations, although exacerbation following allergen exposure is typical of allergic asthma. Although they both show several distinct clinical phenotypes and different biomarkers, there are no ideal biomarkers to stratify asthma phenotypes and guide therapy in clinical practice. Nevertheless, some biomarkers may be helpful to select subsets of atopic patients which might benefit from biologic agents, such as omalizumab. Patients with severe asthma, uncontrolled besides optimal treatment, notwithstanding nonatopic, may also benefit from omalizumab therapy, although currently there are no randomized double-blind placebo controlled clinical trials to support this suggestion. However, omalizumab discontinuation according to each patient's response to therapy and pharmacoeconomical analysis are questions that remain to be answered.
\end{abstract}

\section{Introduction}

Asthma is a heterogeneous disease, usually characterized by chronic airway inflammation. It is defined by the history of respiratory symptoms such as wheeze, shortness of breath, chest tightness, and cough that vary over time and in intensity, together with variable expiratory airflow limitation [1].

The prevalence of asthma, one of the most common chronic diseases in the world [2, 3], has increased during the 1970s and 1980s. Epidemiologic studies from the $90 \mathrm{~s}$ suggested that the prevalence of asthma was around $7.7 \%$ in the United States (US) - over 22 million people-and lethality rate was estimated at 5.2 per 100,000 asthmatic patients per year. Worldwide, 200-300 million people suffer from asthma [1-3], and severe asthma comprises $5-10 \%$ of all asthmatic patients [4]. In Portugal, the prevalence of asthma is estimated to be of $6.8 \%$ [5], affecting around 1 million people. Of these, only $57 \%$ have controlled disease, which means that around 300,000 Portuguese asthmatics need a better intervention to control their disease.

The number of hospitalizations due to asthma was 2,728 in 2016, from a total of 262,229 asthmatic patients registered 
in the Portuguese National Health Service. The standardized mortality rate was, in 2015, of 4.0/100,000 inhabitants for patients above 65 years of age, and of $0.1 / 100,000$ inhabitants for patients below 65 years of age. Nevertheless, and according to the latest Organisation for Economic Co-operation and Development (OECD) report, Portugal is among the countries with less mortality and the country with less hospitalizations due to asthma [6].

The high prevalence of asthma, the impairment of quality of life, the absenteeism, and the large health resources needed to manage this disease makes the economic burden of asthma one of the highest among all chronic diseases. Asthma-related costs have been estimated at up to $2 \%$ of the economic cost of all diseases in developed countries [7]. A recent systematic review examined 68 papers on the economic burden of asthma between 1966 and 2008 and concluded that despite the availability of effective preventive therapies, the cost of asthma treatment has increased significantly over the last few decades [8]. A study conducted in Portugal in 2010 concluded that asthma in adults poses a significant economic burden on the Portuguese healthcare system. Total costs amounted to a grand total of $€ 386,197,211.25$, with direct costs representing $93 \%$ or $€ 359,093,559.82,2.04 \%$ of the total Portuguese healthcare expense in 2010 . The major costs were acute care usage $(30.7 \%)$ and treatment $(37.4 \%)$. A considerable portion of this burden might be eased by improving asthma control in patients, as uncontrolled patients' costs are more than double those of controlled asthma patients [9].

Severe asthma has a heterogeneous definition. The World Health Organization (WHO) suggests that severe asthma includes three groups: (1) untreated asthma; (2) incorrectly treated asthma (as a result of nonadherence, persistent triggers, or comorbidities); and (3) difficult-to-treat asthma. It is also important to distinguish between severe asthma, comprising patients requiring medium/high doses of inhaled corticosteroids in combination with LABA or other controller, and uncontrolled asthma, resulting from inappropriate therapy or persistent problems with adherence or comorbidities [1]. According to the British Guidelines for Asthma, difficult asthma is defined as that with persistent symptoms and/or frequent asthma attacks despite treatment with high-dose therapies or continuous or frequent use of oral steroids [10]. Untreated patients have been recently omitted in the 2014 revision document produced by the task force of the European Respiratory Society (ERS) and the American Thoracic Society (ATS) [11].

Regarding the exposure trigger, generally asthma can be classified as allergic (or atopic) and nonallergic (or nonatopic or intrinsic) asthma. Allergic and nonallergic asthma are distinguished by the presence or absence of clinical allergic reaction and in vitro IgE response to specific aeroallergens $[12,13]$. The triggering of an inflammatory cascade mediated by Immunoglobulin $\mathrm{E}$ (IgE) mast cells' activation, with eosinophils and Th2 lymphocyte synthesis, mobilization, and activation in the airways with IL-4, IL-5, and IL-13 production, leads to bronchial constriction and mucus production with airways narrowing [14-21]. The mechanisms of allergic asthma have been extensively studied with major advances happening in the last two decades. Nonallergic asthma is characterized by its apparent independence from allergen exposure and sensitization, but also by a higher degree of severity $[12,13]$.

Of note, it is important to distinguish nonallergic asthma from aspirin exacerbated respiratory disease (AERD) which also has its own epidemiology, physiopathology, and clinical features: these patients often develop asthma symptoms years after developing rhinitis and nasal polyps due to increased production of cysteinyl-leukotrienes most probably as a result of a polymorphism of the cysteinyl-leukotriene synthase gene [22].

Whether these different clinical subsets of asthma are due to different etiopathogenesis or a different spectrum (or phenotype) of the same disease resulting from different underlying unrecognized mechanisms is still a matter of ongoing debate $[15,23]$.

This review was prepared and discussed by a group of specialists belonging to the Portuguese Network of Severe Asthma Specialists-REAG.

1.1. Allergic versus Nonallergic Asthma. There are similar clinical and physiopathological phenomena between allergic and nonallergic asthma: both can be triggered by exercise, inhaled irritants, or upper airway tract infection; both are associated with rhinitis and both can have higher total serum IgE, airways IgE, airways Th2 cells and Th2, and eosinophilic chemokines and cytokines. Recently, different studies have tried to find a common pathophysiological and immunobiological pattern between both forms of asthma. According to these studies, nonallergic patients may produce the same inflammatory mediators as allergic patients after local IgE production by $\mathrm{T}$ lymphocytes at the bronchial and lung mucosal surface where antigens are presented. This was demonstrated comparing bronchial biopsies samples of nonatopic asthma patients, atopic asthma patients, and nonasthmatic controls [12, 24-27].

Clinically, allergic and nonallergic asthma are virtually indistinguishable during exacerbations, since both lead to signs and symptoms of variable lower airways narrowing and obstruction, which is reversible, at least partially, with bronchodilators [14, 18, 20, 21, 28].

By definition, allergic asthma is clearly associated with allergenic triggering, positive skin prick test, and raised specific $\operatorname{IgE}$ ( $\operatorname{IgE}$ ) $[15,23,29]$. On the other hand, nonallergic asthma is usually of late onset, shows no familial patterns and no genetic trends have been recognized [15, 23, 30], has a higher female prevalence, and tends to be of difficult control and with more severe relapses. A patient with asthma is diagnosed with nonallergic asthma if skin prick tests are negative and no circulating $\operatorname{sIgE}$ are found $[14,18,20,21,28$, 31, 32].

The relationship between allergic and nonallergic asthma prevalence is difficult to ascertain. In some studies, nonallergic asthma prevalence appears to be increasing more than allergic asthma [15]. According to the Swiss Sentinel Surveillance Network (SSSN), the consultations for asthma have decreased over time mainly due to a decrease of allergic asthma. Consultations for nonallergic asthma did not change significantly between 1999 and 2005 [33]. 
TABLE 1: Severe asthma phenotypes proposed by Campo et al. [42].

\begin{tabular}{|c|c|}
\hline Clinical phenotypes & Characteristics \\
\hline $\begin{array}{l}\text { Asthma with frequent severe } \\
\text { exacerbations }\end{array}$ & $\begin{array}{l}\text { Frequent severe exacerbations with periods of relative stability } \\
\text { between exacerbations }\end{array}$ \\
\hline Asthma with fixed airflow obstruction & Irreversible persistent and progressive airflow obstruction \\
\hline Corticosteroid-dependent asthma & $\begin{array}{l}\text { Symptoms cannot be controlled, despite high doses of ICS, and } \\
\text { patients require daily doses of OCS. Reducing the dose of OCS can } \\
\text { often lead to clinical worsening and exacerbations }\end{array}$ \\
\hline \multicolumn{2}{|l|}{ Inflammatory phenotypes } \\
\hline Persistent severe eosinophilic asthma & $\begin{array}{l}\text { Eosinophilia in bronchial biopsies and induced sputum despite high } \\
\text { doses of ICS or OCS. Characterized by more symptoms, lower FEV } \\
\text { values, and more severe exacerbations than the non-eosinophilic } \\
\text { subtype }\end{array}$ \\
\hline $\begin{array}{l}\text { Non-eosinophilic severe asthma with } \\
\text { increased neutrophils }\end{array}$ & $\begin{array}{l}\text { Eosinophils are either absent from the airway or suppressed by } \\
\text { treatment despite the presence of several symptoms, with } \\
\text { inflammation of the airway characterized by an increased percentage } \\
\text { of neutrophils }\end{array}$ \\
\hline Severe paucigranulocytic asthma & $\begin{array}{l}\text { It does not involve inflammation by the classical cell types in the } \\
\text { bronchial biopsy. Inflammation may be located in the distal airway, } \\
\text { which is inaccessible for biopsy, or it may be due to a } \\
\text { bronchiolitis-type disease. No thickening of the subepithelial } \\
\text { basement membrane or signs of classic inflammation are observed. } \\
\text { Other inflammation pathways and other cell types could also be } \\
\text { activated }\end{array}$ \\
\hline
\end{tabular}

ICS: inhaled corticosteroids; OCS: oral corticosteroids.

The true prevalence of severe asthma among nonallergic patients compared to allergic asthma patients is uncertain. Most of the studies assume that severe disease is more prevalent among nonatopic asthma patients. There are conflicting data regarding prevalence trends of asthma and atopy over the last 10-15 years [33]. The proportion of asthmatics with severe disease and a negative skin prick test varies from 17 to $34 \%$ in the Severe Asthma Research Program (SARP) study [34] to $50 \%$ in the ENFUMOSA study [35]. In the ENFUMOSA study, a cross-sectional analysis, it was found that patients with severe asthma were less likely to be skin prickpositive and more likely to have high levels of neutrophils in sputum than patients with less severe asthma [35]. On the other hand, the U-BIOPRED cohort [36] reported a 76.6\% incidence of atopy in severe asthma, including nonsmokers, smokers, and ex-smokers.

Although the prevalence and social and financial burdens of nonallergic asthma seem to be lower than in allergic asthma [19], from a clinical point of view, nonallergic asthma is a true challenge: these patients are usually the most difficult to diagnose, due to their specific epidemiologic features, and the most difficult to treat and control.

1.2. Phenotypes. There is a complex network of different mechanistic and clinical features which are likely linked by a common pattern of reversible respiratory distress associated to distal airways narrowing. In the last decades efforts have focused on the classification of different subsets of asthma patients according to its epidemiology, immunology, biomarkers, response to specific pharmacotherapies, and long-term prognosis. These are broadly called phenotypes: a set of clinical features of a specific genetic pattern in a specific environment. The main goal of the phenotype and endotype philosophy is the development of targeted and personalized pharmacological approaches. Phenotype definition is particularly important in patients with moderate to severe disease and who are not controlled with usual therapy. A detailed and systematic clinical history, including comorbidities, spirometry with bronchodilator test, a skin or blood test panel for sIgE to common regional airborne allergens, and a peripheral blood eosinophil count are very useful for establishing phenotypes. With this information, allergic and nonallergic asthma and eosinophilic or noneosinophilic asthma can be distinguished. This distinction has prognostic and therapeutic implications.

However, although the above-mentioned four phenotypes are considered to be the major ones, research on asthma phenotypes has increased exponentially in the last years and cluster analysis has identified several distinct clinical phenotypes of asthma [34, 37-39]. There is, nonetheless, a clear heterogeneity regarding asthma phenotypes. GINA considers five phenotypes [1] and Wenzel et al. proposed thirteen in 2006 [40]. However, in 2012, these thirteen phenotypes have been reduced to five, due to the evolution towards linking biology to phenotype, namely, at the molecular and genetic levels [41]. In 2013, Campo et al. [42] proposed 6 severe asthma phenotypes subdivided in clinical and inflammatory phenotypes-Table 1 . Smoking is not a phenotype but a disease modifying factor with prognostic implications [42].

1.3. Biomarkers. Several biomarkers have been tested for diagnosis and prediction of clinical response to therapy in asthma, with the aim of achieving personalized therapy. 
Severe asthma is usually characterized by a type 2 disease, associated with atopy and/or eosinophilic inflammation of the airways [43]. However, inflammation in severe asthma is not always characterized by the presence of eosinophils and cytokines of the high-Th2 endotype; in many cases, it may be low-Th2 neutrophilic or low-Th2 paucigranulocytic (type 1 disease) [42].

Currently there are several biomarkers for severe highTh2 asthma, but there is a clear need to identify and select biomarkers of the low-Th2 endotypes. However, this is not an easy task, and several studies in severe asthmatics, such as the ENFUMOSA [35], TENOR [44], SARP [34], and, more recently, the U-BIOPRED [36], have shown a remarkable heterogeneity in the clinical presentation and in the underlying pathophysiological mechanisms of severe asthma.

1.3.1. High-Th2 Endotypes. Although heterogeneous, the classification of the high-Th2 endotypes is mainly based on sputum and systemic eosinophilia [45], and this is considered to be a relevant biomarker. These endotypes also show higher epithelial expression of total IgE $[15,44]$ and Th2 cytokines such as interleukines IL-4, IL-5, and IL-13 [15], two of which, IL-4 and IL13, directly contribute to IgE class switch, thereby increasing IgE [46]. Other known and established biomarkers of Th2 predominant asthma are exaled nitric oxide (FeNO) [47-50] and serum periostin [51]. In a recent study by Busse et al. [52], the authors defined high-Th2 as IgE $\geq 100 \mathrm{IU} / \mathrm{ml}$, eosinophils count $\geq 300 / \mu \mathrm{l}$, and FeNO $\geq 30 \mathrm{ppb}$. Currently, total IgE and serum eosinophils are used not only as disease biomarkers but also as variables on the treatment algorithm of a specific subgroup of severe asthmatic patients who are eligible for anti-IgE omalizumab [53] or anti-IL5 mepolizumab [54]. Indeed, an analysis of biomarkers of the EXTRA study [55] showed that combining biomarkers on the high-Th2 endotypes had therapeutic response implications: patients with severe atopic asthma with high IgE values and Th2 biomarkers (high blood eosinophils and periostin and high FeNO values) showed a better response to omalizumab therapy.

1.3.2. Low-Th2 Endotypes. Although high-Th2 asthma with atopy and eosinophilia is easy to identify, there is no accepted and consensual definition for the low-Th2 endotypes [5658], which comprise around one-third of severe asthmatic patients [59].

Low-Th2 endotypes are currently identified in clinical practice as the absence of biomarkers of atopic asthma and/or eosinophilia. In the majority of cases, the low-Th2 endotypes are defined by the absence of Th 2 inflammatory biomarkers and characterized as neutrophilic inflammation and, less frequently, by paucigranulocytic inflammation $[42,56]$.

Although there is no consensus regarding the percentage of sputum neutrophils that would define the neutrophilic asthma phenotype, some reports mention values between 40 and $70 \%$ [59].

Beyond the sputum leukocyte content, other specific biomarkers that are able to discriminate high-Th2 from low-Th2 are currently under investigation, but are still not applicable in clinical practice.
IL-8 is a cytokine associated with chemotaxis and neutrophilic degranulation and has been found to be elevated in the sputum of patients with severe resistant asthma [60-62]. CXCR1 and CXCR2 have been also found to be elevated in neutrophilic asthma [62]. Other potential biomarkers of neutrophilic asthma are myeloperoxidase [62] and neutrophilic elastase $[61,62]$ that can be assessed in sputum of this subgroup of severe asthmatics.

IL-17 is a biomarker of activation of the Th17 pathway, and correlations between the presence of IL-17 and the level of neutrophils in induced sputum and in circulation have been found in patients with severe asthma $[62,63]$.

There are currently no biomarkers for the subgroup of patients with paucigranulocytic asthma [62]. In this population of patients there is no predominant inflammatory type, and it is possible that other biomarkers of severe asthma, namely, biomarkers of airway remodelling such as osteopontin and angiopoietin, are relevant.

It is necessary to unravel the pathophysiological mechanisms of low-Th2 endotypes in order to identify future biomarkers of these subtypes of asthma $[41,56,62]$.

Currently there are no accurate or precise biomarkers to stratify asthma phenotypes and guide therapy in clinical practice, as illustrated in Figure 1.

1.4. Effect of Interaction of Comorbidities. Uncontrolled allergic rhinitis, gastroesophageal reflux disease (GERD), obesity, vitamin $\mathrm{D}$ deficiency, noncompliance to therapy, and trigger exposure are among the most important effect modifiers of asthma. Of these, due to its prevalence, obesity is one of the most feared comorbidities in asthma patients.

Obese asthma patients show synergy among the two pathologies, i.e., the complexity of the disease is higher than the sum of the diseases, and this interaction worsens the prognosis. Obesity worsens preexisting asthma, through both biochemical and mechanical effects, and potentially impairs response to treatment, and obese patients are more likely to suffer from nonallergic asthma than nonobese patients $[64,65]$.

Even in obese asthmatic patients it seems to be possible to distinguish two different clinical courses based on age of onset and Th2 related biomarkers: early-onset asthma tends to have a more atopic disease, higher IgE, and greater bronchial hyperresponsiveness. These patients seem to have allergic asthma that is complicated by obesity. On the other hand, obese patients with late-onset asthma tend to have less atopy, bronchial hyperresponsiveness, and lower levels of Th2 inflammation. These patients have asthma that has developed in the setting of obesity [66].

\section{Treatment Options for Severe Allergic and Nonallergic Asthma}

The aim of therapy in asthma is achieving disease control. Disease control is considered by the British Thoracic Society [10] as

(i) no daytime symptoms

(ii) no night-time awakening due to asthma 


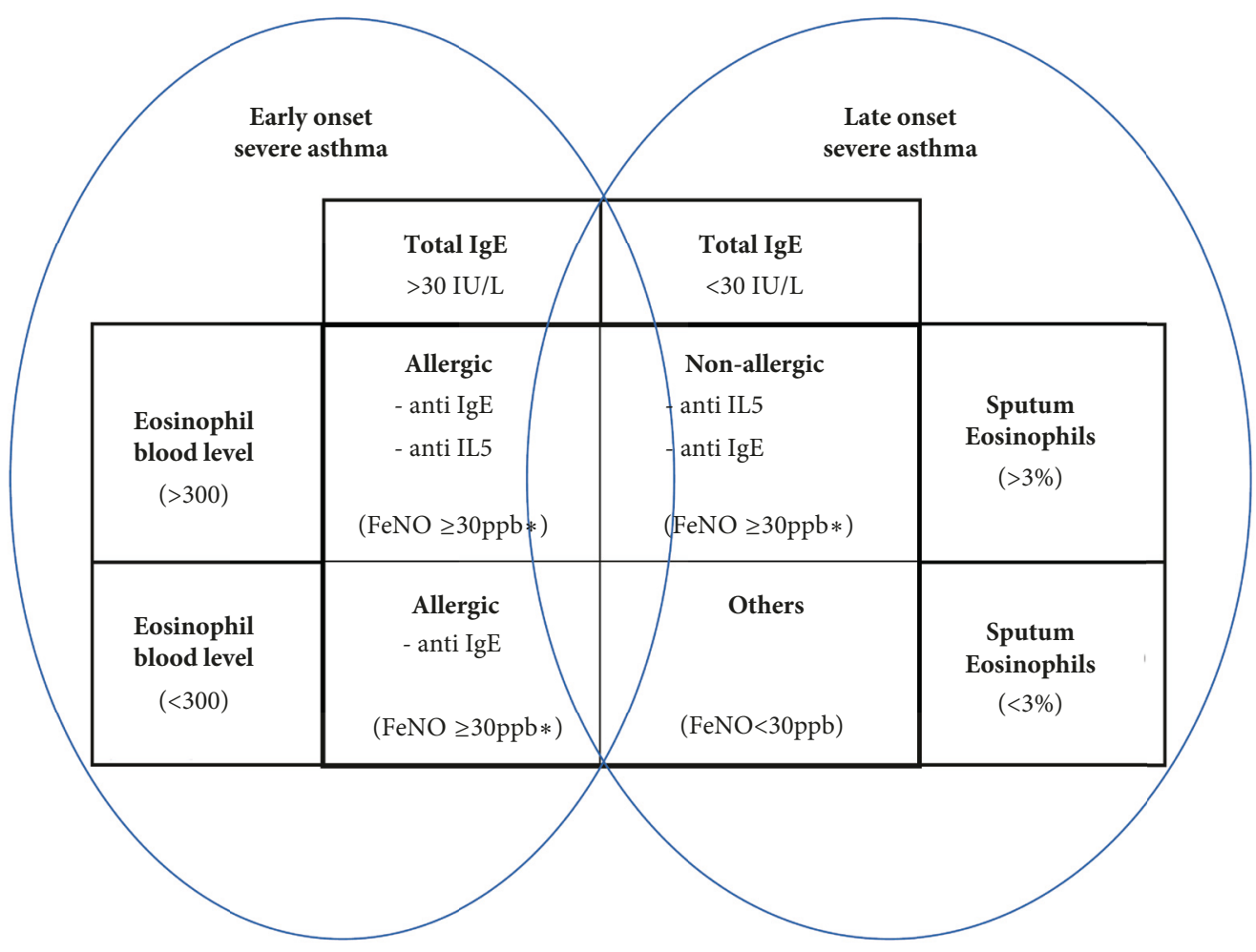

Adapted from [69]

* predictor of good response to anti-IgE treatment

FIGURE 1: Proposed biomarkers to stratify asthma by phenotypes are still not robust enough to guide therapy in clinical practice.

(iii) no need for rescue medication

(iv) no asthma attacks

(v) no limitations on activity including exercise

(vi) normal lung function (in practical terms FEV1 and/or PEF $>80 \%$ predicted or best)

(vii) minimal side effects from medication.

The clinical management of nonallergic asthma is similar to that of allergic asthma. It comprises a combination of nonpharmacological approaches, namely, trigger avoidance and control of comorbidities and pharmacological approaches $[1,10,67]$. Pharmacological approach initiates with ICS as the mainstay of therapy with the addition of LABA if this is insufficient to control symptoms $[1,10,67]$. Additional addon therapy to ICS and LABA according to disease control includes increasing doses of ICS or add-on LAMA, LTRA, or theophylline $[1,10]$. Almost $90 \%$ of asthma patients can generally be controlled with ICS and LABA. Of the remaining $10 \%$, between $17 \%$ and $50 \%$ are nonallergic asthma according to the SARP and ENFUMOSA studies [34, 35]. The UBIOPRED study reported a $30 \%$ incidence of nonatopy in the asthma groups [36].

The presence of comorbidities should prompt the initiation of nonpharmacological and pharmacological strategies towards comorbidities, namely, obesity and GERD.
With the breakthrough of monoclonal antibodies (mAbs) therapies on the verge of the $21^{\text {st }}$ century new pharmacological approaches have been developed and tested in these patients $[24,68]$. Therapy with mAbs is a specific subset of immunotherapy using passive immunity in which preformed antibodies against a target antigen are injected into the body. MAbs can efficiently target an antigen blocking or initiating a biochemical cascade event and through this mechanism achieve a clinical response $[24,68]$. This implies a much higher linkage between pathophysiology, clinical and pharmacotherapy to select the subset of patients who will benefit the most from biological therapy, which revisits phenotypes, immunobiology and endotypes.

2.1. Treatment Options in Severe Allergic Asthma. Sputum analysis and FeNO are very useful in predicting Th2 asthma phenotype, even if no eosinophilia is present. This is of utmost importance to therapeutic strategy definition: allergic asthma with elevated eosinophils and FeNO is more likely to respond to ICS [16] and omalizumab [55]. Allergic Th2 phenotype poorly controlled asthmatic patients should be considered good candidates for omalizumab therapy after add-on ICS/LABA/leukotriene/theophylline therapy [6971].

Omalizumab is a monoclonal antibody designed to bind and inactivate IgE and was approved by EMA in 2009. For patients $\geq 6$ years old omalizumab is indicated as add-on therapy to improve asthma control in patients with severe 
persistent allergic asthma who have a positive skin test or in vitro reactivity to a perennial aeroallergen and frequent daytime symptoms or night-time awakenings and who have had multiple documented severe asthma exacerbations despite daily high-dose inhaled corticosteroids, plus a long-acting inhaled beta2-agonist. For patients $\geq 12$ years of age a reduced lung function $\left(\mathrm{FEV}_{1}<80 \%\right)$ is also required [72].

Omalizumab blocks free serum IgE and limits its binding to the FceRI receptor on the surface of mast cells and basophils. This blockade leads to a reduction in the specific inflammatory response induced by activation of effector cells during the encounter with the allergen [73].

Omalizumab has been also demonstrated to reduce the expression of FceRI on the surface of circulating mast cells and basophils $[74,75]$ which results in a decrease in the release of mediators induced by allergenic stimuli in vitro and in vivo $[74,76,77]$. Omalizumab also seems to intervene in the regulation of the number of circulating basophils which decreases in the treated child [78].

Beyond the anti-IgE mechanism centered on basophils and mast cells, several recent experimental data and clinical observations show that the mechanism of action of omalizumab is more complex than just blocking the allergic response, some of which are mentioned below.

Several studies have shown a decrease in the number of circulating eosinophils and bronchial tissue eosinophils in asthmatics treated with omalizumab [79-82]. Patients with steroid-resistant asthma have been shown to have higher levels of eosinophils, and in these cases omalizumab is a very effective treatment, reducing circulating eosinophils [83]. A proapoptotic effect of omalizumab on eosinophils may contribute to this decrease [84]. Moreover, a study exploring the potential of three biomarkers of Th2-driven inflammation (FeNO, peripheral blood eosinophils, and serum periostin) to predict response to treatment to omalizumab in patients with severe allergic asthma concluded that patients in the highbiomarker subgroup showed a significant decrease in the percentage of exacerbations compared to the low-biomarker subgroup, suggesting that these patients may achieve greater benefit from omalizumab therapy. However, the benefit of such a predictive biomarker of efficacy of omalizumab therapy is currently not established [55].

In a recent study of 673 patients, high levels of periostin and NO exhaled before treatment with omalizumab were associated with a significant decrease in the number of exacerbations [55]. Omalizumab appears to be targeting this Th2 inflammation and a decrease in exhaled NO after treatment has been found in various studies [85]. High levels of these markers prior to initiation of omalizumab have been proposed as biomarkers that predict efficacy with this therapy [55].

Various in vitro, ex vivo, and/or in vivo studies from blood samples, bronchial biopsies, or exhaled air condensates have shown mainly a decrease in the cytokines involved in the recruitment, activation, and survival of eosinophils and IL-5, IL-13, IL-4, IL-8, GM-CSF, eotaxin, RANTES, and the Th2 orientation of the immune response. IFN- $\gamma$, an antiinflammatory cytokine, was not modified in two ex vivo studies after 16 weeks of treatment with omalizumab $[81,86]$.
A modulation of the transcription and/or secretion of these different cytokines could thus contribute to a decrease in the recruitment and activation of the inflammatory cells involved in the late inflammatory stage of asthma and reduce longterm remodelling of the airways [87].

In addition to the above, omalizumab has a preventive effect on viral-induced exacerbations in children with allergic asthma, since blocking $\operatorname{IgE}$ decreases susceptibility to rhinovirus infections and illness [88]. Dendritic cells play a crucial role in innate immune defence against infections, particularly viral infections [89]. During the respiratory allergic response, dendritic cells ensure the presentation of antigens to $\mathrm{T}$ lymphocytes and are also capable of polarizing naive $\mathrm{T}$ lymphocytes in Th2 lymphocytes [90]. Dendritic cells express the FceRI receptor on their surface, such as basophils and mast cells [91]. The binding of IgE to dendritic cells inhibits their antiviral capacities $[92,93]$. A decrease in the expression of FceRI on dendritic cells induced by omalizumab may enhance antiviral immune responses and participate in the prevention of a significant number of asthma exacerbations as demonstrated [88].

\subsection{Treatment Options in Severe Nonallergic Asthma. Pa-} tients with nonallergic asthma are usually more severe and require higher doses of ICS to control symptoms, which may reflect the fact that there may be a degree of corticosteroid resistance as a result of superantigen exposure and activation of MAP kinase pathways [15, 24]. Although patients with severe asthma represent "only" $10 \%$ of asthmatic patients, they are the most challenging and with most impairment of quality of life and absenteeism $[1,8,19]$.

Severe asthma patients with a non-Th2 phenotype with sputum neutrophilia might benefit from macrolide therapy [16]. A very recent study showed that azithromycin reduced asthma exacerbations in both severe eosinophilic and noneosinophilic asthma, suggesting an immunomodulatory effect of macrolides [94]. This immunomodulatory effect may be a possible mechanism of action of omalizumab in both eosinophilic and noneosinophilic asthma. On the other hand, patients with nonallergic but with clear high-Th2 features might be considered good candidates for biotherapies against IL-5, such as mepolizumab or reslizumab [69-71].

In nonallergic asthma, there is frequent elevation of total IgE, including at the bronchial tissue level [95] and it is now established that dendritic cells participate in its pathophysiology $[96,97]$. As in allergic asthma, omalizumab reduces the expression of FceRI on the dendritic cells of nonallergic asthma patients [12]. It is likely that other cells expressing FceRI involved in the pathophysiology of certain nonallergic asthma phenotypes are targeted by omalizumab [98]. Evidence and especially good quality evidence is emerging regarding the efficacy and safety of off-label uses of omalizumab in severe nonallergic asthma [12, 24, 53, 68, 99-106].

The field of action of omalizumab is therefore not limited to a simple anti-IgE activity. The molecule can inflect airway remodelling on one hand and induce clinical efficacy in nonallergic pathologies, but the mechanisms of action at the cellular and cytokine level, anti-Th2 and anti-inflammation, still need to be clarified. In-depth knowledge of the mechanisms 
of action of omalizumab would make it possible to identify predictive biomarkers of efficacy, which are valuable in the phenotyping and therapeutic management of patients with severe asthma.

\section{Conclusions}

Although no good quality evidence is currently available to determine which patients with severe nonatopic asthma should be selected for omalizumab treatment, some issues should always be kept in mind: (a) the diagnosis of nonatopic asthma is not easy and should be carefully confirmed; (b) the definition of severe asthma is heterogeneous and should always be carefully assessed; (c) biomarkers may be helpful to select subsets of patients which might benefit from omalizumab treatment; (d) poor adherence and comorbidities, mainly obesity, interact negatively with asthma and should always be addressed with specific pharmacological and nonpharmacological measures. Based on literature and clinical experience of the authors, there is a clear benefit for allergic asthma patients to be treated with omalizumab. Moreover, those patients with severe nonatopic asthma (including those with high FeNO as a marker of IL-13 inflammation, high eosinophils, and periostin), uncontrolled besides optimal nonpharmacological and pharmacological treatment, may benefit from omalizumab therapy. However, when to suspend omalizumab according to response to therapy in each patient and pharmacoeconomical analysis are questions that remain to be answered.

\section{Disclosure}

Funding for this paper was provided by Novartis Portugal. Funding was used to access all necessary scientific bibliography and cover meeting expenses. Novartis Portugal had no role in the collection, analysis, and interpretation of data, in the writing of the paper, and in the decision to submit the paper for publication.

\section{Conflicts of Interest}

Cláudia Chaves Loureiro reports collaborating and receiving fees from Astra-Zeneca, GlaxoSmithKline, Novartis, Menarini, Mundipharma, Pfizer, and TEVA, through either participation in advisory board or consultancy meetings or congress symposia. Luís Amaral declares collaborating and receiving fees from Novartis. José Alberto Ferreira declares collaborating and receiving fees from Astra-Zeneca, GlaxoSmithKline, Laboratórios Vitória, Novartis, and Sanofi, through either participation in advisory board or consultancy meetings or congress symposia. R. Lima declares collaborating and receiving fees from Astra-Zeneca, Novartis, Mundipharma, GlaxoSmithKline, Menarini, and TEVA, through either participation in advisory board or consultancy meetings or congress symposia. Cecília Pardal declares collaborating and receiving fees from Astra-Zeneca, Novartis, TEVA, Menarini, Mundipharma, Pfizer, Tecnifar, and Bial. Ivone Fernandes declares collaborating and receiving fees from TEVA, Novartis, Menarini, Astra-Zeneca, Novartis, and Tecnifar.
Luisa Semedo declares collaborating and receiving fees from TEVA, Novartis, Menarini, and Pfizer. Ana Maria Arrobas declares collaborating and receiving fees from Astra-Zeneca, Novartis, TEVA, Mundipharma, and Roche, through either participation in advisory board or consultancy meetings.

\section{Acknowledgments}

These authors belong to a subgroup of a larger set of Portuguese experts in severe asthma, GI-IG Grupo de Interesse das Imunoglobulinas, that meet to discuss several issues related to this disease area. In specific, the theme of this paper was exclusively discussed and written only by the authors.

\section{References}

[1] Global Initiative for Asthma (GINA), Global Strategy for Asthma Management and Prevention (2017 update), 2017.

[2] The ISAAC Steering Committee, "Worldwide variation in prevalence of symptoms of asthma, allergic rhinoconjunctivitis, and atopic eczema: ISAAC. The International Study of Asthma and Allergies in Childhood (ISAAC) Steering Committee," Lancet, vol. 351, pp. 1225-1232, 1998.

[3] P. G. J. Burney, C. Luczynska, S. Chinn, and D. Jarvis, "The European Community Respiratory Health Survey," European Respiratory Journal, vol. 7, no. 5, pp. 954-960, 1994.

[4] S. Pakhale, S. Mulpuru, and M. Boyd, "Optimal Management of Severe/Refractory Asthma," Clinical Medicine Insights: Circulatory, Respiratory and Pulmonary Medicine, vol. 5, pp. 37-47, 2011.

[5] A. Sa-Sousa, M. Morais-Almeida, L. F. Azevedo et al., "Prevalence of asthma in Portugal - The Portuguese National Asthma Survey," Clinical and Translational Allergy, vol. 2, article 15, 2012.

[6] Direcção Geral de Saúde, PROGRAMA NACIONAL para as Doenças Respiratórias, 2017.

[7] A. Markham, J. C. Adkins, and B. Jarvis, "Inhaled salmeterol/ fluticasone propionate combination: A pharmacoeconomic review of its use in the management of asthma," PharmacoEconomics, vol. 18, no. 6, pp. 591-608, 2000.

[8] K. Bahadori, M. M. Doyle-Waters, C. Marra et al., "Economic burden of asthma: a systematic review," BMC Pulmonary Medicine, vol. 9, article 24, 2009.

[9] J. P. Barbosa, M. Ferreira-Magalhães, A. Sá-Sousa, L. F. Azevedo, and J. A. Fonseca, "Cost of asthma in Portuguese adults: A population-based, cost-of-illness study," Revista Portuguesa de Pneumologia (English Edition), vol. 23, no. 6, pp. 323-330, 2017.

[10] D. R. James and M. D. Lyttle, "British guideline on the management of asthma: SIGN Clinical Guideline 141, 2014," ADC Education and Practice Edition, vol. 101, no. 6, pp. 319-322, 2016.

[11] K. F. Chung, S. E. Wenzel, J. L. Brozek et al., "International ERS/ATS guidelines on definition, evaluation and treatment of severe asthma," European Respiratory Journal, vol. 43, no. 2, pp. 343-373, 2014

[12] G. Garcia, A. Magnan, R. Chiron et al., "A proof-of-concept, randomized, controlled trial of omalizumab in patients with severe, difficult-to-control, nonatopic asthma," CHEST, vol. 144, no. 2, pp. 411-419, 2013.

[13] A. Nieves, A. Magnan, S. Boniface et al., "Phenotypes of asthma revisited upon the presence of atopy," Respiratory Medicine, vol. 99, no. 3, pp. 347-354, 2005. 
[14] A. S. Amoah, A. G. Forson, and D. A. Boakye, "A review of epidemiological studies of asthma in Ghana.," Ghana Medical Journal, vol. 46, no. 2, pp. 23-28, 2012.

[15] P. J. Barnes, "Intrinsic asthma: not so different from allergic asthma but driven by superantigens?" Clinical \& Experimental Allergy, vol. 39, no. 8, pp. 1145-1151, 2009.

[16] J. Corren, "Asthma phenotypes and endotypes: an evolving paradigm for classification," Discovery Medicine, vol. 15, no. 83, pp. 243-249, 2013.

[17] J. Crane, P. Lampshire, K. Wickens et al., "Asthma, atopy and exhaled nitric oxide in a cohort of 6-yr-old New Zealand children," Pediatric Allergy and Immunology, vol. 23, no. 1, pp. 59-64, 2012.

[18] C. Janson, P. Kalm-Stephens, T. Foucard, K. Alving, and S. L. Nordvall, "Risk factors associated with allergic and non-allergic asthma in adolescents," The Clinical Respiratory Journal, vol. 1, no. 1, pp. 16-22, 2007.

[19] M.-H. Lafeuille, J. Gravel, M. Figliomeni, J. Zhang, and P. Lefebvre, "Burden of illness of patients with allergic asthma versus non-allergic asthma," Journal of Asthma \& Allergy Educators, vol. 50, no. 8, pp. 900-907, 2013.

[20] O. Löwhagen, "Diagnosis of asthma - New theories," Journal of Asthma \& Allergy Educators, vol. 52, no. 6, pp. 538-544, 2015.

[21] S. A. Mahdaviani, S. A. Mohajerani, M. Fakhri et al., "Allergic and nonallergic asthma in children: Are they distinct phenotypes?" Iranian Journal of Allergy, Asthma and Immunology, vol. 13, no. 5, pp. 370-374, 2014.

[22] M. L. Kowalski, "Aspirin-sensitive rhinosinusitis and asthma," The Journal of Allergy and Clinical Immunology, vol. 19, pp. 147175, 2007.

[23] A. L. Comi, A. Tedeschi, M. Lorini, and A. Miadonna, "Novel clinical and serological aspects in non-allergic asthma," Respiratory Medicine, vol. 101, no. 12, pp. 2526-2533, 2007.

[24] C. Domingo, "Omalizumab for severe asthma: Efficacy beyond the atopic patient?" Drugs, vol. 74, no. 5, pp. 521-533, 2014.

[25] M. Humbert, G. Menz, S. Ying et al., "The immunopathology of extrinsic (atopic) and intrinsic (non-atopic) asthma: More similarities than differences," Trends in Immunology, vol. 20, no. 11, pp. 528-533, 1999.

[26] C. E. Owen, "Immunoglobulin E: Role in asthma and allergic disease: Lessons from the clinic," Pharmacology \& Therapeutics, vol. 113, no. 1, pp. 121-133, 2007.

[27] P. D. Mehlhop and K. Blake, "Impact of inadequately controlled asthma: A need for targeted therapy?" Journal of Clinical Pharmacy and Therapeutics, vol. 29, no. 3, pp. 189-194, 2004.

[28] O. Lourenço, A. M. Fonseca, and L. Taborda-Barata, "Demographic, laboratory and clinical characterisation of adult portuguese asthmatic patients," Allergologia et Immunopathologia, vol. 35, no. 5, pp. 177-183, 2007.

[29] B. Leynaert, J. Sunyer, R. Garcia-Esteban et al., "Gender differences in prevalence, diagnosis and incidence of allergic and non-allergic asthma: A population-based cohort," Thorax, vol. 67, no. 7, pp. 625-631, 2012.

[30] M. A. R. Ferreira, M. C. Matheson, D. L. Duffy et al., "Identification of IL6R and chromosome 11q13.5 as risk loci for asthma," The Lancet, vol. 378, no. 9795, pp. 1006-1014, 2011.

[31] C. Ozdemir, B. B. Ceyhan, D. Yazi et al., "Non-atopic asthma in children is related to maternal bronchial hyperreactivity," Pediatric Allergy and Immunology, vol. 19, no. 3, pp. 248-254, 2008.

[32] H. Santoso, "The value of a single skin prick testing for specific IgE Dermatophagoides pteronyssinus to distinguish atopy from non-atopic asthmatic children in the Tropics," Asian Pacific Journal of Allergy and Immunology, vol. 16, no. 2-3, pp. 69-74, 1998.

[33] U. Bollag, L. Grize, and C. Braun-Fahrländer, "Is the ebb of asthma due to the decline of allergic asthma? A prospective consultation-based study by the Swiss Sentinel Surveillance Network, 1999 - 2005," Journal of Family Practice, vol. 26, no. 2, pp. 96-101, 2009.

[34] W. C. Moore, D. A. Meyers, and S. E. Wenzel, "Identification of asthma phenotypes using cluster analysis in the severe asthma research program," American Journal of Respiratory and Critical Care Medicine, vol. 181, no. 4, pp. 315-323, 2010.

[35] European Network for Understanding Mechanisms of Severe Asthma, "The ENFUMOSA cross-sectional European multicentre study of the clinical phenotype of chronic severe asthma. European Network for Understanding Mechanisms of Severe Asthma," European Respiratory Journal, vol. 22, pp. 470-477, 2003.

[36] D. E. Shaw, A. R. Sousa, S. J. Fowler et al., "Clinical and inflammatory characteristics of the European U-BIOPRED adult severe asthma cohort," European Respiratory Journal, vol. 46, no. 5, pp. 1308-1321, 2015.

[37] P. Haldar, I. D. Pavord, D. E. Shaw et al., "Cluster analysis and clinical asthma phenotypes," American Journal of Respiratory and Critical Care Medicine, vol. 178, no. 3, pp. 218-224, 2008.

[38] V. Siroux and J. Garcia-Aymerich, "The investigation of asthma phenotypes," Current Opinion in Allergy and Clinical Immunology, vol. 11, no. 5, pp. 393-399, 2011.

[39] C. Loureiro, P. Sa-Couto, A. Todo-Bom, and J. Bousquet, "Cluster analysis in phenotyping a Portuguese population," Revista Portuguesa de Pneumologia (English Edition), vol. 21, no. 6, pp. 299-306, 2015.

[40] S. E. Wenzel, "Asthma: defining of the persistent adult phenotypes," The Lancet, vol. 368, no. 9537, pp. 804-813, 2006.

[41] S. E. Wenzel, "Asthma phenotypes: the evolution from clinical to molecular approaches," Nature Medicine, vol. 18, no. 5, pp. 716-725, 2012.

[42] P. Campo, F. Rodriguez, S. Sanchez-Garcia et al., "Phenotypes and endotypes of uncontrolled severe asthma: new treatments," Journal of Investigational Allergology and Clinical Immunology, vol. 23, pp. 76-88, 2013.

[43] J. V. Fahy, "Type 2 inflammation in asthma-present in most, absent in many," Nature Reviews Immunology, vol. 15, no. 1, pp. 57-65, 2015.

[44] B. E. Chipps, R. S. Zeiger, L. Borish et al., "Key findings and clinical implications from the Epidemiology and Natural History of Asthma: Outcomes and Treatment Regimens (TENOR) study," The Journal of Allergy and Clinical Immunology, vol. 130, no. 2, pp. 332-342.e10, 2012.

[45] I. H. van Veen, A. Ten Brinke, S. A. Gauw, P. J. Sterk, K. F. Rabe, and E. H. Bel, "Consistency of sputum eosinophilia in difficultto-treat asthma: a 5-year follow-up study," The Journal of Allergy and Clinical Immunology, vol. 124, no. 3, pp. 615-617.e2, 2009.

[46] L. Cameron, Q. Hamid, E. Wright et al., "Local synthesis of epsilon germline gene transcripts, IL-4, and IL-13 in allergic nasal mucosa after ex vivo allergen exposure," The Journal of Allergy and Clinical Immunology, vol. 106, no. 1 I, pp. 46-52, 2000.

[47] A. D. Smith, J. O. Cowan, K. P. Brassett et al., "Exhaled nitric oxide: A predictor of steroid response," American Journal of Respiratory and Critical Care Medicine, vol. 172, no. 4, pp. 453459, 2005. 
[48] L. M. Van Den Toorn, S. E. Overbeek, J. C. De Jongste et al., "Airway inflammation is present during clinical remission of atopic asthma," American Journal of Respiratory and Critical Care Medicine, vol. 164, no. 11, pp. 2107-2113, 2001.

[49] S. L. Jones, J. Kittelson, J. O. Cowan et al., “The predictive value of exhaled nitric oxide measurements in assessing changes in asthma control," American Journal of Respiratory and Critical Care Medicine, vol. 164, no. 5, pp. 738-743, 2001.

[50] K. Chibana, J. B. Trudeau, A. T. Mustovich et al., "IL-13 induced increases in nitrite levels are primarily driven by increases in inducible nitric oxide synthase as compared with effects on arginases in human primary bronchial epithelial cells," Clinical \& Experimental Allergy, vol. 38, no. 8, pp. 936-946, 2008.

[51] J. Corren, R. F. Lemanske Jr., N. A. Hanania et al., "Lebrikizumab treatment in adults with asthma," The New England Journal of Medicine, vol. 365, no. 12, pp. 1088-1098, 2011.

[52] W. W. Busse, S. T. Holgate, S. W. Wenzel et al., "Biomarker Profiles in Asthma With High vs Low Airway Reversibility and Poor Disease Control," CHEST, vol. 148, no. 6, pp. 1489-1496, 2015.

[53] A. Navinés-Ferrer, E. Serrano-Candelas, G.-J. Molina-Molina, and M. Martín, "IgE-Related Chronic Diseases and Anti-IgEBased Treatments," Journal of Immunology Research, vol. 2016, Article ID 8163803, 12 pages, 2016.

[54] H. G. Ortega, S. W. Yancey, B. Mayer et al., "Severe eosinophilic asthma treated with mepolizumab stratified by baseline eosinophil thresholds: a secondary analysis of the DREAM and MENSA studies," The Lancet Respiratory Medicine, vol. 4, no. 7, pp. 549-556, 2016.

[55] N. A. Hanania, S. Wenzel, K. Rosén et al., "Exploring the effects of omalizumab in allergic asthma: an analysis of biomarkers in the EXTRA study," American Journal of Respiratory and Critical Care Medicine, vol. 187, no. 8, pp. 804-811, 2013.

[56] K. Samitas, E. Zervas, and M. Gaga, "T2-low asthma: Current approach to diagnosis and therapy," Current Opinion in Pulmonary Medicine, vol. 23, no. 1, pp. 48-55, 2017.

[57] P. J. Sterk and R. Lutter, "Asthma phenotyping: TH2-high, TH2-low, and beyond," The Journal of Allergy and Clinical Immunology, vol. 133, no. 2, pp. 395-396, 2014.

[58] C.-H. S. Kuo, S. Pavlidis, M. Loza et al., "T-helper cell type 2 (Th2) and non-Th2 molecular phenotypes of asthma using sputum transcriptomics in U-BIOPRED," European Respiratory Journal, vol. 49, no. 2, 2017.

[59] F. Schleich, G. Brusselle, R. Louis et al., "Heterogeneity of phenotypes in severe asthmatics. The Belgian Severe Asthma Registry (BSAR)," Respiratory Medicine, vol. 108, no. 12, pp. 17231732, 2014.

[60] B. J. Green, S. Wiriyachaiporn, C. Grainge et al., "Potentially pathogenic airway bacteria and neutrophilic inflammation in treatment resistant severe asthma," PLOS ONE, vol. 9, no. 6, Article ID e100645, 2014.

[61] L. G. Wood, K. J. Baines, J. Fu, H. A. Scott, and P. G. Gibson, "The neutrophilic inflammatory phenotype is associated with systemic inflammation in asthma," CHEST, vol. 142, no. 1, pp. 86-93, 2012.

[62] F. Schleich, D. Sophie, and L. Renaud, "Biomarkers in the management of difficult asthma," Current Topics in Medicinal Chemistry, vol. 16, no. 14, pp. 1561-1573, 2016.

[63] I. Agache, C. Ciobanu, C. Agache, and M. Anghel, "Increased serum IL-17 is an independent risk factor for severe asthma," Respiratory Medicine, vol. 104, no. 8, pp. 1131-1137, 2010.
[64] L. Garcia-Marcos, A. A. Pena, R. Busquets-Monge et al., "How the presence of rhinoconjunctivitis and the severity of asthma modify the relationship between obesity and asthma in children 6-7 years old," Clinical \& Experimental Allergy, vol. 38, no. 7, pp. 1174-1178, 2008.

[65] S. Pradeepan, G. Garrison, and A. E. Dixon, "Obesity in asthma: approaches to treatment," Current Allergy and Asthma Reports, vol. 13, no. 5, pp. 434-442, 2013.

[66] E. R. Sutherland, E. Goleva, T. S. King et al., "Cluster analysis of obesity and asthma phenotypes," PLOS ONE, vol. 7, no. 5, Article ID e36631, 2012.

[67] A. B. Becker and E. M. Abrams, "Asthma guidelines: The global initiative for asthma in relation to national guidelines," Current Opinion in Allergy and Clinical Immunology, vol. 17, no. 2, pp. 99-103, 2017.

[68] C. Domingo, X. Pomares, N. Angril, N. Rudi, M. J. Amengual, and R. M. Mirapeix, "Effectiveness of omalizumab in nonallergic severe asthma," Journal of Biological Regulators and Homeostatic Agents, vol. 27, no. 1, pp. 45-53, 2013.

[69] A. Froidure, J. Mouthuy, S. R. Durham, P. Chanez, Y. Sibille, and C. Pilette, "Asthma phenotypes and IgE responses," European Respiratory Journal, vol. 47, no. 1, pp. 304-319, 2016.

[70] I. D. Pavord, S. Korn, P. Howarth et al., "Mepolizumab for severe eosinophilic asthma (DREAM): a multicentre, double-blind, placebo-controlled trial," The Lancet, vol. 380, no. 9842, pp. 651659, 2012

[71] M. Castro, S. Mathur, F. Hargreave et al., "Reslizumab for poorly controlled, eosinophilic asthma: a randomized, placebocontrolled study," American Journal of Respiratory and Critical Care Medicine, vol. 184, no. 10, pp. 1125-1132, 2011.

[72] Summary of Product Characteristics Omalizumab. Approved by EMA 2009, Last updated 2016.

[73] L. C. Presta, S. J. Lahr, R. L. Shields et al., "Humanization of an antibody directed against IgE," The Journal of Immunology, vol. 151 , no. 5, pp. 2623-2632, 1993.

[74] L. A. Beck, G. V. Marcotte, D. MacGlashan Jr., A. Togias, and S. Saini, "Omalizumab-induced reductions in mast cell Fce psilon RI expression and function," The Journal of Allergy and Clinical Immunology, vol. 114, no. 3, pp. 527-530, 2004.

[75] H. Lin, K. M. Boesel, D. T. Griffith et al., "Omalizumab rapidly decreases nasal allergic response and FcepsilonRI on basophils," The Journal of Allergy and Clinical Immunology, vol. 113, no. 2, pp. 297-302, 2004.

[76] O. Noga, G. Hanf, and G. Kunkel, "Immunological and clinical changes in allergic asthmatics following treatment with omalizumab," International Archives of Allergy and Immunology, vol. 131, no. 1, pp. 46-52, 2003.

[77] J. A. Eckman, P. M. Sterba, D. Kelly et al., "Effects of omalizumab on basophil and mast cell responses using an intranasal cat allergen challenge," The Journal of Allergy and Clinical Immunology, vol. 125, no. 4, pp. 889.e7-895.e7, 2010.

[78] D. A. Hill, M. C. Siracusa, K. R. Ruymann, E. D. Tait Wojno, D. Artis, and J. M. Spergel, "Omalizumab therapy is associated with reduced circulating basophil populations in asthmatic children," Allergy: European Journal of Allergy and Clinical Immunology, vol. 69, no. 5, pp. 674-677, 2014.

[79] R. Djukanović, S. J. Wilson, M. Kraft et al., "Effects of treatment with anti-immunoglobulin $\mathrm{E}$ antibody omalizumab on airway inflammation in allergic asthma," American Journal of Respiratory and Critical Care Medicine, vol. 170, no. 6, pp. 583-893, 2004 . 
[80] M. Massanari, H. Nelson, T. Casale et al., "Effect of pretreatment with omalizumab on the tolerability of specific immunotherapy in allergic asthma," The Journal of Allergy and Clinical Immunology, vol. 125, no. 2, pp. 383-389, 2010.

[81] Y. Takaku, T. Soma, F. Nishihara et al., “Omalizumab attenuates airway inflammation and interleukin- 5 production by mononuclear cells in patients with severe allergic asthma," International Archives of Allergy and Immunology, vol. 161, pp. 107-117, 2013.

[82] M. Massanari, S. T. Holgate, W. W. Busse, P. Jimenez, F. Kianifard, and R. Zeldin, "Effect of omalizumab on peripheral blood eosinophilia in allergic asthma," Respiratory Medicine, vol. 104, no. 2, pp. 188-196, 2010.

[83] I. Kupryś-Lipińska, K. Molińska, and P. Kuna, "effect of omalizumab on eosinophilic inflammation of the respiratory tract in patients with allergic asthma," Pneumonologia i Alergologia Polska, vol. 84, no. 4, pp. 232-243, 2016.

[84] O. Noga, G. Hanf, I. Brachmann et al., "Effect of omalizumab treatment on peripheral eosinophil and T-lymphocyte function in patients with allergic asthma," The Journal of Allergy and Clinical Immunology, vol. 117, no. 6, pp. 1493-1499, 2006.

[85] Y.-C. Huang, B. Leyko, and M. Frieri, "Effects of omalizumab and budesonide on markers of inflammation in human bronchial epithelial cells," Annals of Allergy, Asthma \& Immunology, vol. 95, no. 5, pp. 443-451, 2005.

[86] J. T. Schroeder, A. P. Bieneman, K. L. Chichester et al., "Decreases in human dendritic cell-dependent TH2-like responses after acute in vivo IgE neutralization," The Journal of Allergy and Clinical Immunology, vol. 125, no. 4, pp. 896-901.e6, 2010.

[87] S. Holgate, N. Smith, M. Massanari, and P. Jimenez, "Effects of omalizumab on markers of inflammation in patients with allergic asthma," Allergy, vol. 64, no. 12, pp. 1728-1736, 2009.

[88] A. Esquivel, W. W. Busse, A. Calatroni et al., "Effects of Omalizumab on Rhinovirus Infections, Illnesses, and exacerbations of asthma," American Journal of Respiratory and Critical Care Medicine, vol. 196, no. 8, pp. 985-992, 2017.

[89] M. Lommatzsch, K. Bratke, A. Bier et al., "Airway dendritic cell phenotypes in inflammatory diseases of the human lung," European Respiratory Journal, vol. 30, pp. 878-886, 2007.

[90] K. Shortman and S. H. Naik, "Steady-state and inflammatory dendritic-cell development," Nature Reviews Immunology, vol. 7, no. 1, pp. 19-30, 2007.

[91] T. Bieber, H. D. L. Salle, A. Wollenberg et al., "Human epidermal Langerhans cells express the high affinity receptor for immunoglobulin E (Fc epsilon RI)," The Journal of Experimental Medicine, vol. 175, no. 5, pp. 1285-1290, 1992.

[92] J. T. Schroeder, A. P. Bieneman, H. Xiao et al., “TLR9-and FceRI-mediated responses oppose one another in plasmacytoid dendritic cells by down-regulating receptor expression," The Journal of Immunology, vol. 175, no. 9, pp. 5724-5731, 2005.

[93] J. R. Tversky, T. V. Le, A. P. Bieneman, K. L. Chichester, R. G. Hamilton, and J. T. Schroeder, "Human blood dendritic cells from allergic subjects have impaired capacity to produce interferon- $\alpha$ via toll-like receptor 9," Clinical \& Experimental Allergy, vol. 38, no. 5, pp. 781-788, 2008.

[94] P. G. Gibson, I. A. Yang, J. W. Upham et al., "Effect of azithromycin on asthma exacerbations and quality of life in adults with persistent uncontrolled asthma (AMAZES): a randomised, double-blind, placebo-controlled trial," The Lancet, vol. 390, no. 10095, pp. 659-668, 2017.

[95] P. Takhar, C. J. Corrigan, L. Smurthwaite et al., "Class switch recombination to $\operatorname{IgE}$ in the bronchial mucosa of atopic and nonatopic patients with asthma," The Journal of Allergy and Clinical Immunology, vol. 119, no. 1, pp. 213-218, 2007.

[96] J. P. Lynch, S. B. Mazzone, M. J. Rogers et al., "The plasmacytoid dendritic cell: at the cross-roads in asthma," European Respiratory Journal, vol. 43, no. 1, pp. 264-275, 2014.

[97] P. Stoll, A. Bähker, M. Ulrich et al., "The dendritic cell high-affinity IgE receptor is overexpressed both in asthma and severe COPD," Clinical \& Experimental Allergy, 2015.

[98] M. Humbert, J. A. Grant, L. Taborda-Barata et al., "Highaffinity IgE receptor (FcepsilonRI)-bearing cells in bronchial biopsies from atopic and nonatopic asthma," American Journal of Respiratory and Critical Care Medicine, vol. 153, no. 6, pp. 1931-1937, 1996.

[99] M. Van Den Berge, R. G. Pauw, J. G. R. De Monchy, C. A. Van Minnen, D. S. Postma, and H. A. M. Kerstjens, "Beneficial effects of treatment with anti-IgE antibodies (omalizumab) in a patient with severe asthma and negative skin-prick test results," CHEST, vol. 139, no. 1, pp. 190-193, 2011.

[100] F. Menzella, R. Piro, N. Facciolongo, C. Castagnetti, A. Simonazzi, and L. Zucchi, "Long-term benefits of omalizumab in a patient with severe non-allergic asthma," Allergy, Asthma \& Clinical Immunology, vol. 7, article 9, 2011.

[101] J. R. Stokes and T. B. Casale, "The use of anti-IgE therapy beyond allergic asthma," Journal of Allergy and Clinical Immunology: In Practice, vol. 3, no. 2, pp. 162-166, 2015.

[102] M. Lommatzsch, S. Korn, R. Buhl, and J. C. Virchow, "Against all odds: Anti-IgE for intrinsic asthma?” Thorax, vol. 69, no. 1, pp. 94-96, 2014.

[103] L. P. de Llano, M. D. C. Vennera, F. J. Álvarez et al., "Effects of omalizumab in non-atopic asthma: results from a Spanish multicenter registry," The Journal of Asthma, vol. 50, no. 3, pp. 296-301, 2013.

[104] K. S. Babu, R. Polosa, and J. B. Morjaria, "Anti-IgE-emerging opportunities for Omalizumab," Expert Opinion on Biological Therapy, vol. 13, no. 5, pp. 765-777, 2013.

[105] D. El-Qutob, "Off-Label Uses of Omalizumab," Clinical Reviews in Allergy \& Immunology, vol. 50, no. 1, pp. 84-96, 2016.

[106] C. Sattler, G. Garcia, and M. Humbert, "Novel targets of omalizumab in asthma," Current Opinion in Pulmonary Medicine, vol. 23, no. 1, pp. 56-61, 2017. 


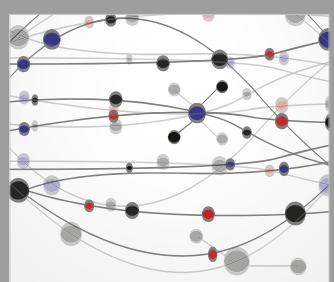

The Scientific World Journal
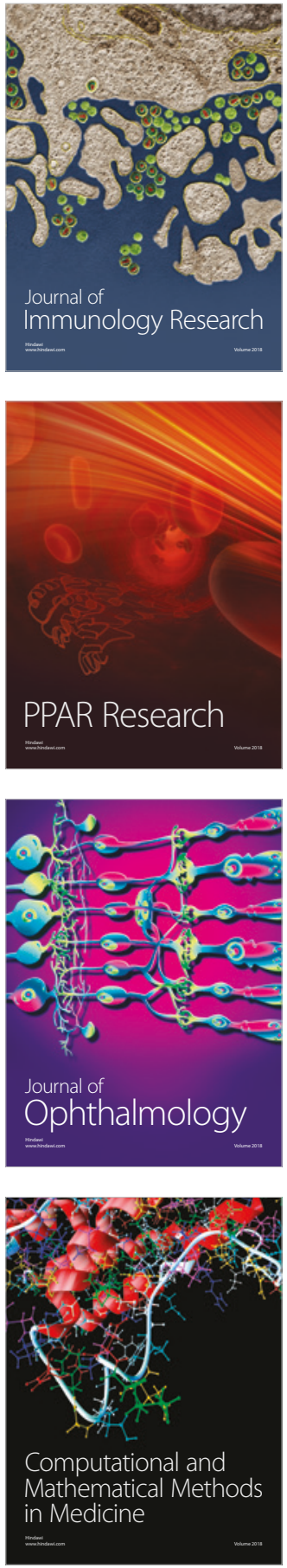

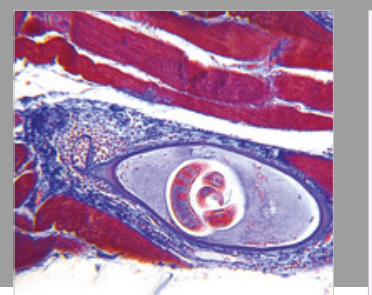

Gastroenterology Research and Practice

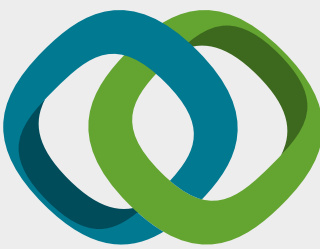

\section{Hindawi}

Submit your manuscripts at

www.hindawi.com
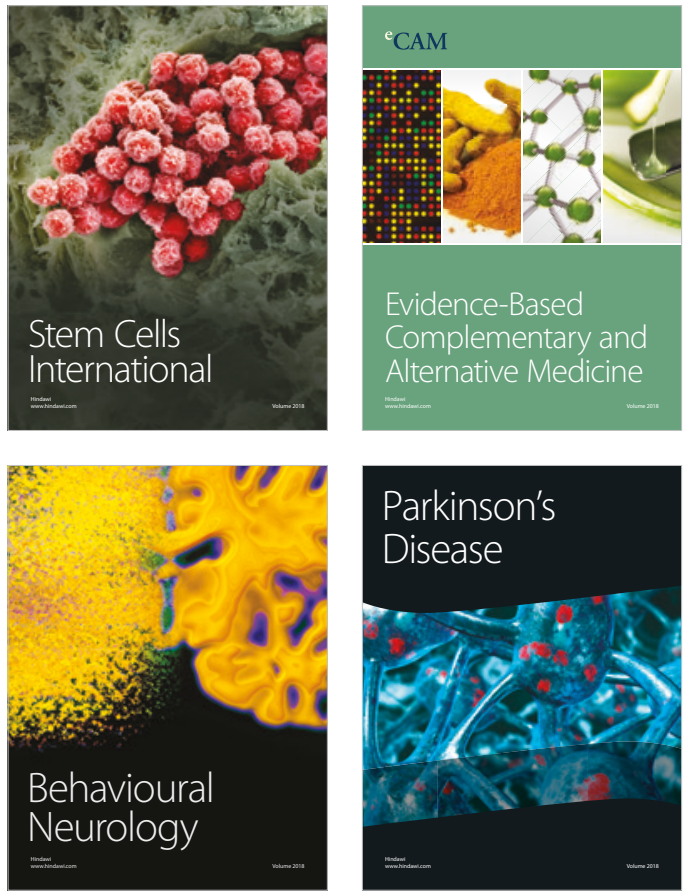

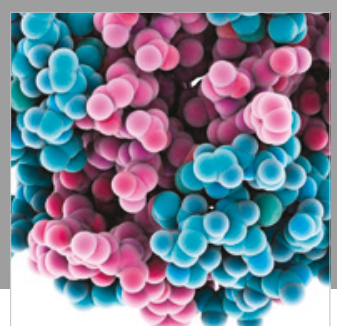

ournal of

Diabetes Research

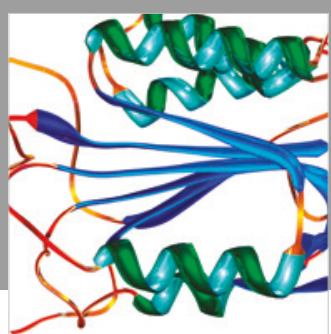

Disease Markers
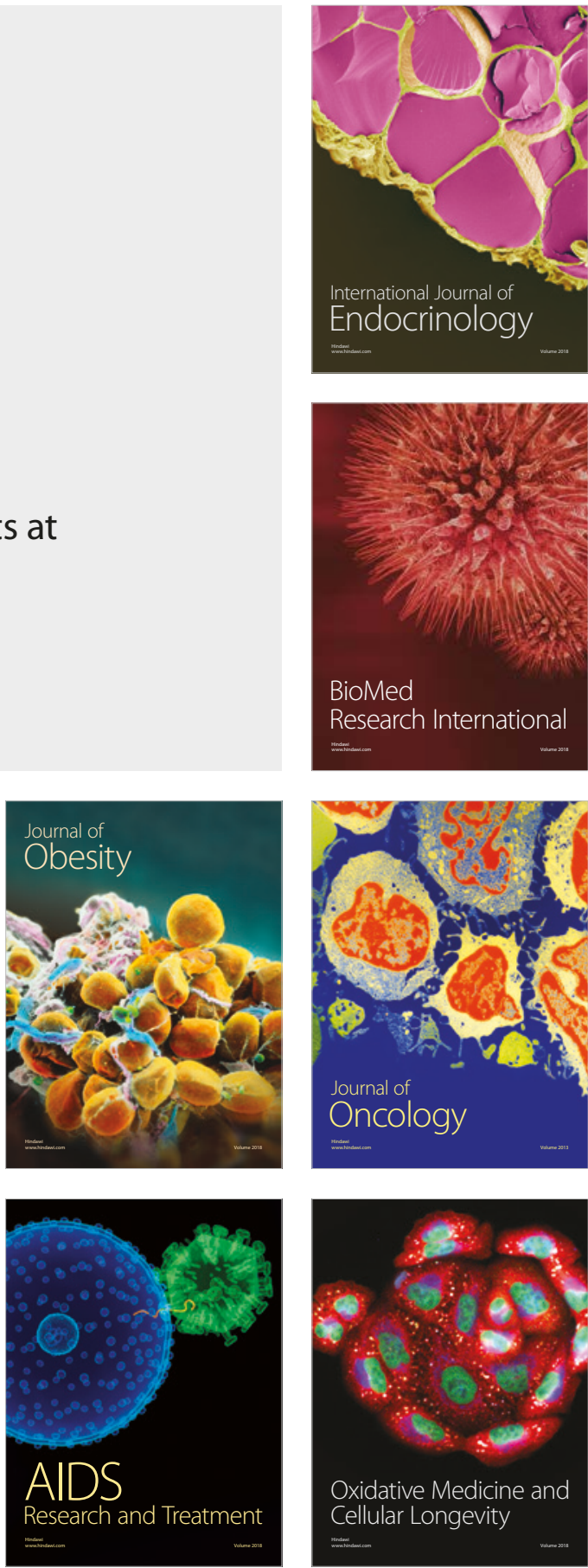